\title{
Possible Underlying Mechanisms Responsible for Aldosterone and Mineralocorticoid Receptor-Dependent Renal Injury
}

\author{
Hideyasu Kiyomoto ${ }^{1}$, Kazi Rafiq ${ }^{2}$, Mahbub Mostofa ${ }^{3}$, and Akira Nishiyama ${ }^{2} *$ \\ ${ }^{I}$ Department of CardioRenal and Cerebrovascular Medicine, and ${ }^{2}$ Department of Pharmacology, \\ Faculty of Medicine, Kagawa University, 1750-1 Ikenobe, Miki-cho, Kita-gun, Kagawa 761-0793, Japan \\ ${ }^{3}$ Department of Pharmacology, Bangladesh Agricultural University, Mymensingh, Bangladesh
}

Received August 3, 2008

\begin{abstract}
There is increasing evidence indicating the roles of aldosterone and mineralocorticoid receptor (MR) in the pathogenesis of renal injury. In rats, chronic treatment with aldosterone and salt results in severe proteinuria and renal tissue injury, characterized by glomerulosclerosis and tubulointerstitial fibrosis. Aldosterone-induced renal tissue injury is associated with increases in reactive oxygen species (ROS) levels and activation of mitogen-activated protein kinases (MAPKs) or Rho-kinase. Treatment with a selective MR antagonist, eplerenone, prevents aldosterone-induced increases in ROS levels and MAPK activity and ameliorates renal injury. In vitro studies have revealed that MR is highly expressed in glomerular mesangial cells (RMCs), podocytes, and renal interstitial fibroblasts. In these renal cells, aldosterone induces cellular injury through NADPH oxidase-dependent ROS production and activation of MAPKs or Rhokinase. Such aldosterone-induced renal cellular injury is markedly attenuated by treatment with eplerenone. These data suggest that aldosterone induces renal injury via activation of MR through mechanisms that cannot be simply explained by changes in blood pressure. In this review, we summarized recent findings on the roles of aldosterone and MR in the pathogenesis of renal injury with particular emphasis on potential underlying mechanisms.
\end{abstract}

Keywords: aldosterone, mineralocorticoid receptor, reactive oxygen species (ROS), extracellular signal-regulated kinases (ERK) 1/2, eplerenone, kidney

\section{Introduction}

Aldosterone is recognized as a steroid hormone that regulates body fluid homeostasis $(1,2)$; however, accumulating evidence suggests that aldosterone is a key factor in mediating cardiovascular injury (3). Patients with primary aldosteronism have a higher incidence of cardiovascular complications than do patients with essential hypertension (4). The Randomized Aldactone Evaluation Study (RALES) revealed that adding a non-specific mineralocorticoid receptor (MR) antagonist, spironolactone, to standard therapy, including

*Corresponding author. akira@kms.ac.jp Published online in J-STAGE on December 5, 2008 (in advance) doi: $10.1254 /$ jphs.08R02CR

Invited article angiotensin-converting enzyme (ACE) inhibitors, significantly reduces morbidity and mortality in patients with moderate to severe heart failure (5). More recently, the Eplerenone Post-Acute Myocardial Infarction Heart Failure Efficacy and Survival Study (EPHESUS) showed that adding a selective MR antagonist, eplerenone, to optimal medical therapy reduces morbidity and mortality among patients with acute myocardial infarction complicated by left ventricular dysfunction and heart failure (6). Based on the results from these two multicenter clinical analyses and other clinical studies $(7,8)$, most national guideline groups now recommended MR antagonists in preference to other antihypertensive agents in hypertensive patients with heart diseases $(9-11)$. However, recent studies have also indicated the potential roles of aldosterone and MR in the pathogenesis of renal injury. For example, patients with primary aldosteronism have higher incidence of 
proteinuria or albuminuria than do patients with essential hypertension $(4,12,13)$. Plasma aldosterone levels are positively correlated with urinary protein excretion levels and negatively correlated with glomerular filtration rate in patients with primary aldosteronism (14), chronic kidney disease (CKD), and diabetic nephropathy (15). Furthermore, addition of MR antagonists to either ACE inhibitors or angiotensin-receptor blockers (ARBs) reduces albuminuria in patients with type 2 diabetic nephropathy (16) or CKD $(17-19)$. In this review, we have summarized recent evidence regarding the roles of aldosterone and MR in the pathogenesis of renal injury with particular emphasis on their possible underlying mechanisms.

\section{Localization of MR in the kidney}

It has been shown that chronic administration of aldosterone with salt loading to rats elicits proteinuria, glomerular mesangial injury, and tubulointerstitial fibrosis $(20,21)$. Since these aldosterone-induced renal injuries were prevented by treatment with MR antagonists, it is possible that aldosterone directly induces renal tissue injury via activation of locally expressed MR. Based on the observations in aldosterone-infused rats $(20-24)$, we investigated the existence of MR in cultured rat mesangial cells (RMCs) (25) and renal fibroblasts (26) by Western blotting and real-time RT-PCR. An MR-specific antibody detected significant MR protein expression in RMCs and renal fibroblasts with a band of approximately $110-120 \mathrm{kDa}$, which corresponds to the molecular weight of rat MR in tissues $(25,26)$. We also investigated the subcellular localization of MR in RMCs (25). In fluorolabeling experiments using confocal microscopy, MR protein expression was observed in both the cytoplasm and nuclear fraction with barely detectable levels in the membrane fraction (Fig. 1A). These observations were consistent with those by Terada et al. (27) that MR translocation from the cytoplasm to the nucleus is induced by treatment with aldosterone in RMCs. Recently, Nishikawa et al. (28) have reported that human glomerular mesangial cells produced aldosterone via steroidogenic enzymes such as $\mathrm{P} 450 \mathrm{scc}, 3 \beta$-hydroxysteroid dehydrogenase, 21hydroxylase, and CYP11B2. Therefore, it seems likely that locally produced aldosterone stimulates the transcription rates of mineralocorticoid-responsive genes by activating MR in mesangial cells (28). MR expression was also detected in podocytes $(24,29)$ and renal tubular cells $(20,30,31)$. Taken together, MR is localized not only in distal tubular cells but also in other renal cells, which mediate aldosterone-induced renal tissue injury.

\section{Renal tissue injury induced by chronic infusion of aldosterone and its possible mechanisms}

Chronic administration of aldosterone with salt supplement to uninephrectomized $(24,32-35)$ or nonnephrectomized (23) rats caused hypertension with proteinuria and podocyte abnormality (Fig. 1B). Aldosterone treatment also induces severe glomerular injury and tubulointersitial fibrosis (23) (Fig. 1C). Increases in multiple factors, including MAPKs (23), Rho-kinase (35), plasminogen activator inhibitor-1 $(36-38)$, transforming growth factor- $\beta 1(33,35,36)$, connective tissue growth factor $(33,35)$, proinflammatory cytokines such as osteopontin $(32,36)$ and monocyte chemoattractant protein-1 $(32,35)$, and reactive oxygen species (ROS) $(23,24)$, have also been observed in renal tissues of aldosterone-infused rats. Among those factors, ROS production may be an important mediator of aldosterone-induced renal injury $(23,24)$. We have shown that aldosterone/salt-induced renal injury is associated with increases in thiobarbituric acid reactive substances (TBARS) contents, a marker of ROS production (Fig. 2A), and in mRNA levels of NADPH oxidase components, p22phox, Nox-4, and gp91phox in renal cortical tissue (23). Treatment with eplerenone suppressed aldosterone-induced increases in TBARS levels (Fig. 2A) and NADPH oxidase expression. Interestingly, tempol, a superoxide dismutase mimetic, normalized renal cortical TBARS levels (Fig. 2A) and prevented progression of proteinuria and renal tissue injury (Fig. 1C) in these animals $(21,23)$. These data suggest that ROS production is essential in the progression of renal injury induced by aldosterone.

Shibata et al. (24) showed that chronic infusion of aldosterone with high-salt treatment induced hypertension with massive proteinuria and glomerular podocyte injury in uninephrectomized rats. They also showed that glomerular expressions of nephrin and podocin, slit diaphragm-associated molecules, were markedly reduced, whereas expression of desmin, a damaged podocyte marker (39), was upregulated in uninephrectomized aldosterone-infused rats. Treatment with tempol or eplerenone significantly reduced oxidative stress markers and attenuated podocyte damage and proteinuria in aldosterone-infused rats (24). Moreover, both tempol and eplerenone have been shown to markedly attenuate salt-induced podocyte injury and proteinuria in other rodent models of hypertensive glomerulosclerosis $(29,40,41)$. These findings suggest that aldosterone-induced podocyte injury underlies the pathogenesis of proteinuria, possibly through MR activation and ROS production $(20,21)$.

We have shown that in aldosterone-infused rats, 
A

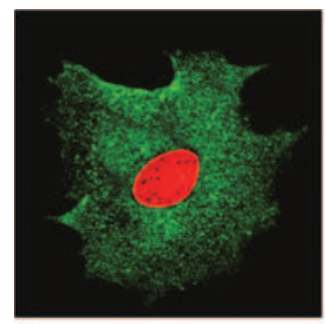

B



$1 \% \mathrm{NaCl}$

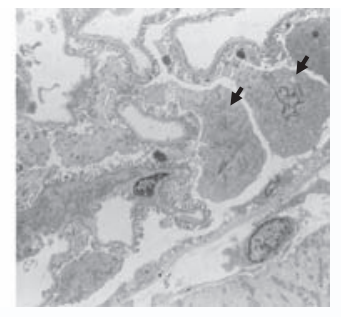

$1 \% \mathrm{NaCl}$

+ aldosterone

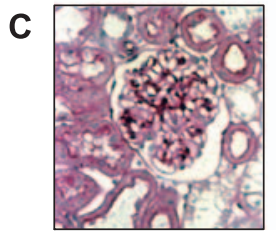

vehicle

$+1 \% \mathrm{NaCl}$

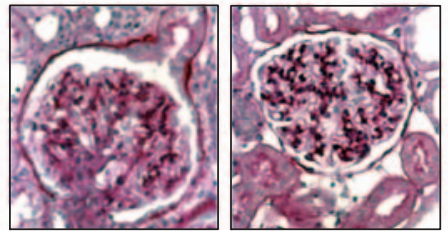

$1 \% \mathrm{NaCl}+$ aldosterone

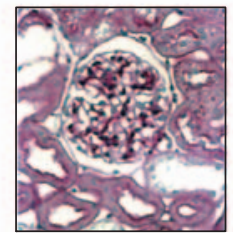

$1 \% \mathrm{NaCl}$

+ aldosterone

+ eplerenone

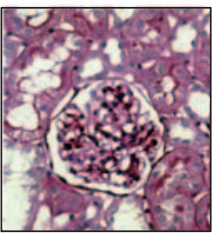

$1 \% \mathrm{NaCl}$

+ aldosterone

+ tempol

Fig. 1. Localization of mineralocorticoid receptor (MR) and aldosterone-induced renal injury in rats. A: Distribution of mineralocorticoid receptor (green) and $\alpha 1$-integrin (red) staining in cultured rat mesangial cells (RMCs). Superimposition of the image does not reveal any areas of co-localization of MR and $\alpha 1$-integrin (known as a membrane marker), indicating a lack of expression of MR in the cell membrane of RMC. Data are modified from Ref. 25 with permission. B: Glomerular histological findings for podocyte ultrastructure (original magnification, $\times 3000$ ). Rats treated with aldosterone $/ 1 \% \mathrm{NaCl}$ exhibit podocyte abnormality (arrow). C: Photomicrographs of glomeruli (periodic acid-Schiff stain, original magnification $\times 400$ ). Rats treated with aldosterone $/ 1 \% \mathrm{NaCl}$ exhibit glomerular sclerosis. Treatment with eplerenone or tempol markedly ameliorates the changes induced by aldosterone $/ 1 \% \mathrm{NaCl}$. Data are from Ref. 23 with permission.

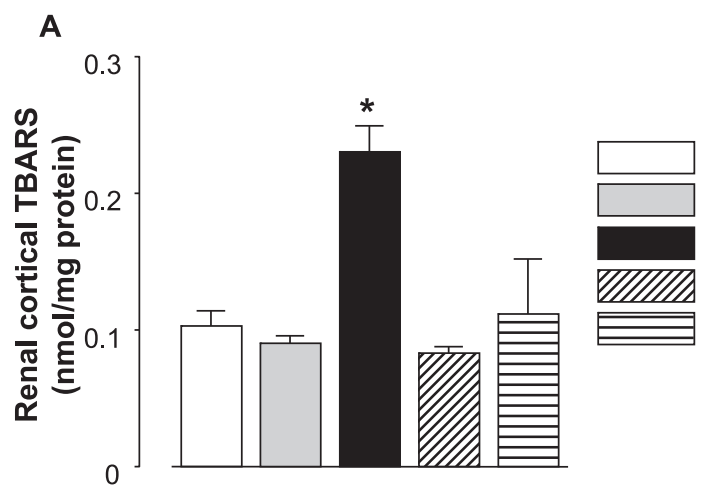

vehicle

$1 \% \mathrm{NaCl}$

$1 \% \mathrm{NaCl}+$ aldosterone

$1 \% \mathrm{NaCl}+$ aldosterone + eplerenone

$1 \% \mathrm{NaCl}+$ aldosterone + tempol

B

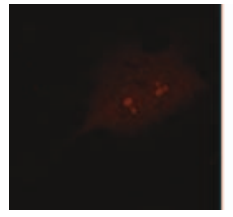

control

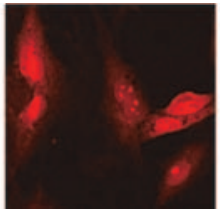

aldosterone

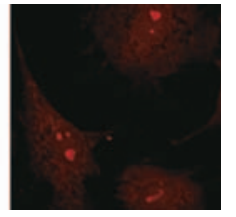

eplrerenone

$$
+
$$

aldosterone

Fig. 2. Effects of aldosterone on reactive oxygen species production in rats and RMCs. A: Thiobarbituric acid reactive substances (TBARS) contents in renal cortical tissues. Rats treated with aldosterone $/ 1 \% \mathrm{NaCl}$ show increases in TBARS levels in renal cortical tissues. Treatment with eplerenone or tempol markedly prevents aldosterone-induced increases in TBARS levels. ${ }^{*} P<0.05 v s$ vehicle-treated rats. Data are from Ref. 23 with permission. B: Effects of aldosterone on dihydroethidium staining in RMCs. Eplerenone $(10 \mu \mathrm{mol} / \mathrm{L})$ significantly attenuated aldosterone-induced $(100 \mathrm{nmol} / \mathrm{L}$ for $3 \mathrm{~h})$ increases in dihydroethidium staining. Original magnification, $\times 1600$. Data are modified from Ref. 43 with permission. 




B


Fig. 3. Effects of aldosterone and eplerenone on NADPH oxidase activity and membranous translocation of p47phox and p67phox in RMCs. A: Effects of aldosterone on NADPH oxidase activity in RMCs. Eplerenone $(10 \mu \mathrm{mol} / \mathrm{L}) \quad$ significantly attenuated aldosterone $(100 \mathrm{nmol} / \mathrm{L})$-induced increases in NADPH oxidase activity. B: Effects of aldosterone on membranous translocation of p47phox and p67phox in RMC. Eplerenone significantly attenuated aldosterone-induced membranous translocation of p47phox and p67phox. $V=$ vehicle. Data are modified from Ref. 43 with permission. renal injury is associated with activation of MAPKs: extracellular signal-regulated kinase (ERK) $1 / 2$, c-Jun $\mathrm{N}$-terminal, and big MAPK-1 in renal cortical tissues, but not p38 MAPK (23). These data suggest the MAPKs are involved in aldosterone-dependent renal injury. However, effects of specific inhibition of MAPKs have not been examined in aldosterone-infused rats. The small GTP-binding protein RhoA has recently been proposed as another candidate for mediating hypertensive glomerulosclerosis $(35,42)$. We demonstrated that a specific Rho-kinase inhibitor, fasudil, attenuated aldosterone-induced TGF- $\beta$ activation and ameliorated the progression of renal injury in rats (35). Further studies are needed to clarify the precise molecular mechanisms of aldosterone/MR-induced renal injury.

\section{Renal cell injury induced by aldosterone and its possible mechanisms}

As mentioned above, renal injury in aldosteroneinfused rats is associated with increases in NADPH expression and ROS levels (23). The finding that aldosterone-induced renal injury was attenuated by treatment with tempol indicates that ROS are an important mediator of aldosterone-dependent renal injury $(23,24)$. These observations prompted us to investigate whether aldosterone and MR have direct effects on NADPH oxidase activation and superoxide anion $\left(\mathrm{O}_{2}^{-}\right)$generation in cultured RMCs. We found that aldosterone directly stimulates $\mathrm{O}_{2}^{-}$generation in RMCs (Fig. 2B). Aldosterone-induced $\mathrm{O}_{2}^{-}$production was accompanied by an increase in NADPH oxidase activity (Fig. 3A) and translocation of p47phox and p67phox, cytosol components of NADPH oxidase, to the RMC membrane (Fig. 3B) (43). These findings are consistent with the hypothesis that aldosterone stimulates ROS generation through NADPH oxidase-dependent mechanisms (21). Recent studies show that aldosterone induces mesangial cell apoptosis and that administration of antioxidants, free radical scavengers, or MR blockers partially attenuates the proapoptotic effects of aldosterone (22).

In rats, aldosterone-induced renal injury is associated with increased activity of ERK1/2 (23) and Rho-kinase (35). In cultured mesangial cells $(25,27,44)$ and renal fibroblasts (26), aldosterone activates ERK1/2, and its activation is reversed by treatment with spironolactone or eprelenone. We confirmed that eprelenone or the inhibition of the ERK1/2 cascade with PD98059 abolishes aldosterone-induced cell proliferation and deformability in RMCs (25) and collagen synthesis in renal fibroblasts (26). These data support the possible contributions of MAPKs to aldosterone-induced renal cell injury. However, the specific relationship between 
A





control $\mathrm{V} \quad \mathrm{CH}$

aldosterone
B

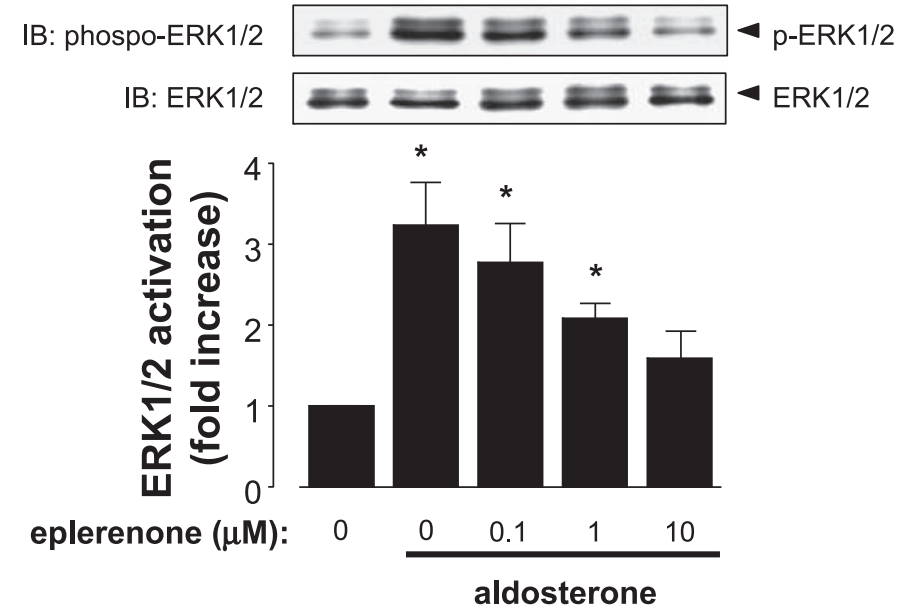

Fig. 4. Effects of aldosterone and eplerenon on extracellular signal-regulated kinase (ERK) $1 / 2$ phosphorylation in RMCs. A: Effects of actinomycin D (AD) and cycloheximide $(\mathrm{CH})$ on aldosterone (100 nmol/L)-induced ERK 1/2 phosphorylation in RMCs. Aldosterone-induced ERK1/2 phosphorylation is not altered by pretreatment with $\mathrm{AD}(5 \mu \mathrm{g} / \mathrm{mL})$ or $\mathrm{CH}$ $(10 \mu \mathrm{g} / \mathrm{mL}) . \mathrm{V}=$ vehicle. Data are modified from Ref. 25 with permission. B: Effects of eplerenone on aldosterone-induced ERK 1/2 phosphorylation in RMCs. Results were normalized by arbitrarily setting the densitometry of vehicle-treated cells to 1.0. Aldosterone-induced ERK1/2 phosphorylation is dosedependently inhibited by treatment with eplerenone. $* P<0.05$ vs control $(0 \mathrm{nmol} / \mathrm{L}$ of aldosterone $)$. Data are from Ref. 25 with permission.
ROS and MAPKs in mediating renal cell injury remains unknown. Furthermore, the role of Rho-kinase in aldosterone-induced renal cell injury is under investigation. The roles of other factors including transforming growth factor- $\beta$, plasminogen activator inhibitor type 1 , and osteopontin in mediating renal fibroblast proliferation, collagen synthesis, or matrix degradation have also been indicated by recent studies $(45,46)$.

In addition to its classical genomic actions mediated through the regulation of nuclear gene transcription and protein synthesis, aldosterone elicits rapid responses probably via the non-genomic pathways in a variety of cells $(47-50)$. Rapid activation of ERK1/2 was observed with a peak at $5-10$ min after stimulation of aldosterone in RMCs (25) and renal fibroblasts (26). We also found that aldosterone activated ERK $1 / 2$ in both RMCs and renal fibroblasts under the condition where de novo synthesis of proteins was inhibited by treatment with actinomycin D and/or cycloheximide (Fig. 4A) $(25,26)$. These data suggest the involvement of non-genomic mechanisms in aldosterone-induced activation of signal transduction pathways in renal cells. The rapid effect of alsosterone on ERK1/2 activation was attenuated by treatment with eplerenone in a dosedependent manner (Fig. 4B). MR antagonists also block aldosterone-induced activation of ERK $1 / 2$ in vascular smooth muscle cells (51) and Chinese hamster ovary cells transfected with human MR (52). On the other hand, Terada et al. (27) showed that aldosterone-induced ERK1/2 activation in RMCs was attenuated by inhibition of genomic pathways. It has also been shown that spironolactone had no inhibitory effects on rapid activation of ERK $1 / 2$ induced by aldosterone in cortical collecting duct cells (50). There is no satisfactory explanation for the discrepancies between these observations. However, these findings combined with the data obtained from animal experiments (23) indicate that ERK $1 / 2$ is an important mediator of aldosteroneinduced renal cellular injury.

\section{Conclusions}

In this review, we discussed the potential roles of aldosterone and MR in the pathogenesis of renal injury. Both in vitro and in vivo experiments indicate that aldosterone and MR contribute to the progression of renal injury via direct actions on tubulointerstitial fibroblasts, glomerular mesangial cells, and podcytes. Molecular mechanisms including those involving ROS, MAPKs, and Rho-kinase may be involved in aldosterone /MR-induced renal injury. However, further studies are needed to determine the precise mechanisms of aldosterone- and MR-mediated renal cell injury, including the contributions of genomic and non-genomic pathways. 


\section{References}

1 Simpson SA, Tait JF, Wettstein A, Neher R, Von Euw J, Reichstein $\mathrm{T}$. Isolation from the adrenals of a new crystalline hormone with especially high effectiveness on mineral metabolism. Experientia. 1953;9:333-335.

2 Williams JS, Williams GH. 50th anniversary of aldosterone. J Clin Endocrinol Metab. 2003;88:2364-2372.

3 Fuller PJ, Young MJ. Mechanisms of mineralocorticoid action. Hypertension. 2005;46:1227-1235.

4 Nishimura M, Uzu T, Fujii T, Kuroda S, Nakamura S, Inenaga $\mathrm{T}$, et al. Cardiovascular complications in patients with primary aldosteronism. Am J Kidney Dis. 1999;33:261-266.

5 Pitt B, Zannad F, Remme WJ, Cody R, Castaigne A, Perez A, et al. The effect of spironolactone on morbidity and mortality in patients with severe heart failure. Randomized Aldactone Evaluation Study Investigators. N Engl J Med. 1999;341:709717.

6 Pitt B, Remme W, Zannad F, Neaton J, Martinez F, Roniker B, et al. Eplerenone, a selective aldosterone blocker, in patients with left ventricular dysfunction after myocardial infarction. $\mathrm{N}$ Engl J Med. 2003;348:1309-1321.

7 Pitt B, White H, Nicolau J, Martinez F, Gheorghiade M, Aschermann M, et al. Eplerenone reduces mortality 30 days after randomization following acute myocardial infarction in patients with left ventricular systolic dysfunction and heart failure. J Am Coll Cardiol. 2005;46:425-431.

8 Weintraub WS, Zhang Z, Mahoney EM, Kolm P, Spertus JA, Caro J, et al. Cost-effectiveness of eplerenone compared with placebo in patients with myocardial infarction complicated by left ventricular dysfunction and heart failure. Circulation. 2005; 111:1106-1113.

9 Japanese Society of Hypertension guidelines for the management of hypertension (JSH 2004). Hypertens Res. 2006;29 Suppl:S1-S105.

10 Buse JB, Ginsberg HN, Bakris GL, Clark NG, Costa F, Eckel R, et al. Primary prevention of cardiovascular diseases in people with diabetes mellitus: a scientific statement from the American Heart Association and the American Diabetes Association. Circulation. 2007;115:114-126.

11 Mancia G, De Backer G, Dominiczak A, Cifkova R, Fagard R, Germano G, et al. 2007 Guidelines for the Management of Arterial Hypertension: The Task Force for the Management of Arterial Hypertension of the European Society of Hypertension (ESH) and of the European Society of Cardiology (ESC). J Hypertens. 2007;25:1105-1187.

12 Rossi GP, Bernini G, Desideri G, Fabris B, Ferri C, Giacchetti $\mathrm{G}$, et al. Renal damage in primary aldosteronism: results of the PAPY Study. Hypertension. 2006;48:232-238.

13 Sechi LA, Novello M, Lapenna R, Baroselli S, Nadalini E, Colussi GL, et al. Long-term renal outcomes in patients with primary aldosteronism. JAMA. 2006;295:2638-2645.

14 Catena C, Colussi G, Nadalini E, Chiuch A, Baroselli S, Lapenna R, et al. Relationships of plasma renin levels with renal function in patients with primary aldosteronism. Clin J Am Soc Nephrol. 2007;2:722-731.

15 Schjoedt KJ, Andersen S, Rossing P, Tarnow L, Parving HH. Aldosterone escape during blockade of the renin-angiotensinaldosterone system in diabetic nephropathy is associated with enhanced decline in glomerular filtration rate. Diabetologia.
2004;47:1936-1939.

16 Sato A, Hayashi K, Naruse M, Saruta T. Effectiveness of aldosterone blockade in patients with diabetic nephropathy. Hypertension. 2003;41:64-68.

17 Bomback AS, Kshirsagar AV, Amamoo MA, Klemmer PJ. Change in proteinuria after adding aldosterone blockers to ACE inhibitors or angiotensin receptor blockers in CKD: a systematic review. Am J Kidney Dis. 2008;51:199-211.

18 Chrysostomou A, Pedagogos E, MacGregor L, Becker GJ. Double-blind, placebo-controlled study on the effect of the aldosterone receptor antagonist spironolactone in patients who have persistent proteinuria and are on long-term angiotensinconverting enzyme inhibitor therapy, with or without an angiotensin II receptor blocker. Clin J Am Soc Nephrol. 2006;1:256-262.

19 Sato A, Hayashi K, Saruta T. Antiproteinuric effects of mineralocorticoid receptor blockade in patients with chronic renal disease. Am J Hypertens. 2005;18:44-49.

20 Nagase M, Fujita T. Aldosterone and glomerular podocyte injury. Clin Exp Nephrol. 2008;12:233-242.

21 Nishiyama A, Abe Y. Molecular mechanisms and therapeutic strategies of chronic renal injury: renoprotective effects of aldosterone blockade. J Pharmacol Sci. 2006;100:9-16.

22 Mathew JT, Patni H, Chaudhary AN, Liang W, Gupta A, Chander $\mathrm{PN}$, et al. Aldosterone Induces Mesangial Cell Apoptosis both in vivo and in vitro. Am J Physiol Renal Physiol. 2008;295:F73-F81.

23 Nishiyama A, Yao L, Nagai Y, Miyata K, Yoshizumi M, Kagami S, et al. Possible contributions of reactive oxygen species and mitogen-activated protein kinase to renal injury in aldosterone/salt-induced hypertensive rats. Hypertension. 2004; 43:841-848.

24 Shibata S, Nagase M, Yoshida S, Kawachi H, Fujita T. Podocyte as the target for aldosterone: roles of oxidative stress and Sgk1. Hypertension. 2007;49:355-364.

25 Nishiyama A, Yao L, Fan Y, Kyaw M, Kataoka N, Hashimoto $\mathrm{K}$, et al. Involvement of aldosterone and mineralocorticoid receptors in rat mesangial cell proliferation and deformability. Hypertension. 2005;45:710-716.

26 Nagai Y, Miyata K, Sun GP, Rahman M, Kimura S, Miyatake A, et al. Aldosterone stimulates collagen gene expression and synthesis via activation of ERK1/2 in rat renal fibroblasts. Hypertension. 2005;46:1039-1045.

27 Terada Y, Kobayashi T, Kuwana H, Tanaka H, Inoshita S, Kuwahara $\mathrm{M}$, et al. Aldosterone stimulates proliferation of mesangial cells by activating mitogen-activated protein kinase 1/2, cyclin D1, and cyclin A. J Am Soc Nephrol. 2005;16:22962305 .

28 Nishikawa T, Suematsu S, Saito J, Soyama A, Ito H, Kino T, et al. Human renal mesangial cells produce aldosterone in response to low-density lipoprotein (LDL). J Steroid Biochem Mol Biol. 2005;96:309-316.

29 Nagase M, Yoshida S, Shibata S, Nagase T, Gotoda T, Ando K, et al. Enhanced aldosterone signaling in the early nephropathy of rats with metabolic syndrome: possible contribution of fatderived factors. J Am Soc Nephrol. 2006;17:3438-3446.

30 Quinkler M, Zehnder D, Eardley KS, Lepenies J, Howie AJ, Hughes SV, et al. Increased expression of mineralocorticoid effector mechanisms in kidney biopsies of patients with heavy proteinuria. Circulation. 2005;112:1435-1443. 
31 Suh JM, Yu CT, Tang K, Tanaka T, Kodama T, Tsai MJ, et al. The expression profiles of nuclear receptors in the developing and adult kidney. Mol Endocrinol. 2006;20:3412-3420.

32 Blasi ER, Rocha R, Rudolph AE, Blomme EA, Polly ML, McMahon EG. Aldosterone/salt induces renal inflammation and fibrosis in hypertensive rats. Kidney Int. 2003;63:1791-1800.

33 Fan YY, Baba R, Nagai Y, Miyatake A, Hosomi N, Kimura S, et al. Augmentation of intrarenal angiotensin II levels in uninephrectomized aldosterone/salt-treated hypertensive rats; renoprotective effects of an ultrahigh dose of olmesartan. Hypertens Res. 2006;29:169-178.

34 Peng H, Carretero OA, Raij L, Yang F, Kapke A, Rhaleb NE. Antifibrotic effects of N-acetyl-seryl-aspartyl-Lysyl-proline on the heart and kidney in aldosterone-salt hypertensive rats. Hypertension. 2001;37:794-800.

35 Sun GP, Kohno M, Guo P, Nagai Y, Miyata K, Fan YY, et al. Involvements of Rho-kinase and TGF-beta pathways in aldosterone-induced renal injury. J Am Soc Nephrol. 2006;17: 2193-2201.

36 Chun TY, Chander P, Kim JW, Pratt JH, Stier CT. Aldosterone, but not angiotensin II, increased profibrotic factors in the kidney of adrenalectomized stroke prone spontaneously hypertensive rats. Am J Physiol Endocrinol Metab. 2008;295:E305-E312.

37 Ma J, Weisberg A, Griffin JP, Vaughan DE, Fogo AB, Brown NJ. Plasminogen activator inhibitor-1 deficiency protects against aldosterone-induced glomerular injury. Kidney Int. 2006;69: 1064-1072.

38 Yuan J, Jia R, Bao Y. Aldosterone up-regulates production of plasminogen activator inhibitor-1 by renal mesangial cells. J Biochem Mol Biol. 2007;40:180-188.

39 Yaoita E, Kawasaki K, Yamamoto T, Kihara I. Variable expression of desmin in rat glomerular epithelial cells. Am J Pathol. 1990;136:899-908.

40 Nagase M, Matsui H, Shibata S, Gotoda T, Fujita T. Saltinduced nephropathy in obese spontaneously hypertensive rats via paradoxical activation of the mineralocorticoid receptor: role of oxidative stress. Hypertension. 2007;50:877-883.

41 Nagase M, Shibata S, Yoshida S, Nagase T, Gotoda T, Fujita T. Podocyte injury underlies the glomerulopathy of Dahl salthypertensive rats and is reversed by aldosterone blocker. Hypertension. 2006;47:1084-1093.

42 Nishikimi T, Akimoto K, Wang X, Mori Y, Tadokoro K,
Ishikawa Y, et al. Fasudil, a Rho-kinase inhibitor, attenuates glomerulosclerosis in Dahl salt-sensitive rats. J Hypertens. 2004;22:1787-1796.

43 Miyata K, Rahman M, Shokoji T, Nagai Y, Zhang GX, Sun GP, et al. Aldosterone stimulates reactive oxygen species production through activation of NADPH oxidase in rat mesangial cells. J Am Soc Nephrol. 2005;16:2906-2912.

44 Otani H, Otsuka F, Inagaki K, Takeda M, Miyoshi T, Suzuki J, et al. Antagonistic effects of bone morphogenetic protein-4 and -7 on renal mesangial cell proliferation induced by aldosterone through MAPK activation. Am J Physiol Renal Physiol. 2007;292:F1513-F1525.

45 Huang W, Xu C, Kahng KW, Noble NA, Border WA, Huang Y. Aldosterone and TGF- $\beta_{1}$ synergistically increase PAI-1 and decrease matrix degradation in rat renal mesangial and fibroblast cells. Am J Physiol Renal Physiol. 2008;294:F1287-F1295.

46 Irita J, Okura T, Kurata M, Miyoshi K, Fukuoka T, Higaki J. Osteopontin in rat renal fibroblasts: functional properties and transcriptional regulation by aldosterone. Hypertension. 2008; 51:507-513.

47 Funder JW. The nongenomic actions of aldosterone. Endocr Rev. 2005;26:313-321.

48 Good DW, George T, Watts BA 3rd. Aldosterone potentiates 1,25-dihydroxyvitamin D3 action in renal thick ascending limb via a nongenomic, ERK-dependent pathway. Am J Physiol Cell Physiol. 2003;285:C1122-C1130.

49 Mazak I, Fiebeler A, Muller DN, Park JK, Shagdarsuren E, Lindschau C, et al. Aldosterone potentiates angiotensin IIinduced signaling in vascular smooth muscle cells. Circulation. 2004;109:2792-2800.

50 Rossol-Haseroth K, Zhou Q, Braun S, Boldyreff B, Falkenstein E, Wehling $\mathrm{M}$, et al. Mineralocorticoid receptor antagonists do not block rapid ERK activation by aldosterone. Biochem Biophys Res Commun. 2004;318:281-288.

51 Ishizawa K, Izawa Y, Ito H, Miki C, Miyata K, Fujita Y, et al. Aldosterone stimulates vascular smooth muscle cell proliferation via big mitogen-activated protein kinase 1 activation. Hypertension. 2005;46:1046-1052.

52 Grossmann C, Benesic A, Krug AW, Freudinger R, Mildenberger $\mathrm{S}$, Gassner B, et al. Human mineralocorticoid receptor expression renders cells responsive for nongenotropic aldosterone actions. Mol Endocrinol. 2005;19:1697-1710. 